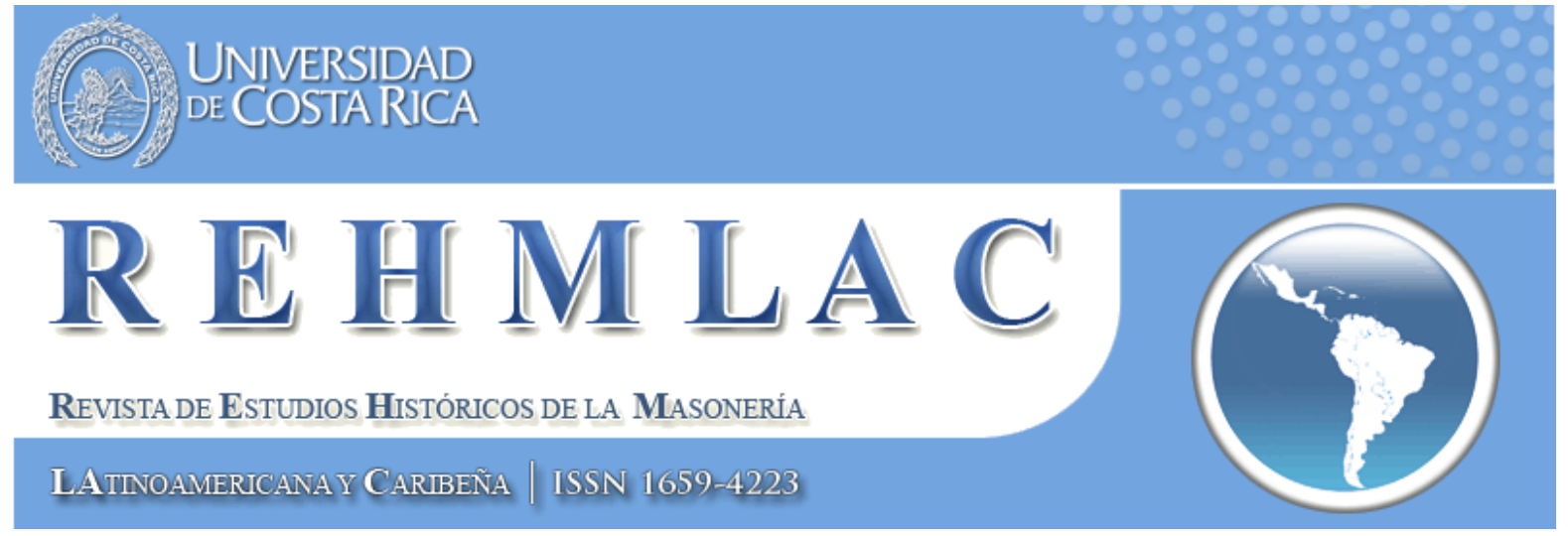

Esteban Sánchez Solano

"La participación político-partidista de la Iglesia:

EI Partido Unión Católica y sus estrategias de movilización política en el marco del conflicto entre la Iglesia Católica y el Estado liberal en Costa Rica (1889-1898)"
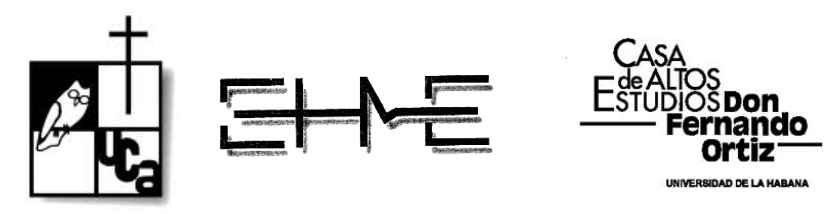
(C) Esteban Sánchez Solano y REHMLAC.

\section{Equipo REHMLAC}

Consejo Científico:

Miguel Guzmán-Stein (Universidad de Costa Rica, Costa Rica)

José Antonio Ferrer Benimeli (Universidad de Zaragoza, España)

Margaret Jacob (University of California Los Angeles, United States)

Eduardo Torres Cuevas (Universidad de La Habana, Cuba)

María Eugenia Vázquez Semadeni (Stanford University, United States)

Éric Saunier (Université du Havre, France)

Andreas Önnerfors (Lunds universitet, Sverige)

Samuel Sánchez Gálvez (Universidad Carlos Rafael Rodríguez de Cienfuegos, Cuba)

Roberto Valdés Valle (Universidad Centroamericana “José Simeón Cañas”, El Salvador)

Céline Sala (Université de Perpignan, France)

Dominique Soucy (Université de Franche-Comté, France)

Guillermo de los Reyes Heredia (University of Houston, United States)

Dévrig Mollès (Université de Strasbourg, France)

Felipe Santiago del Solar Guajardo (Universidad ARCIS, Santiago de Chile)

Carlos Francisco Martínez Moreno (Universidad Nacional Autónoma de México, México)

Michel Goulart da Silva (Universidade do Estado de Santa Catarina, Brasil)

Correctores de estilo:

Sylvia Hottinger (Centro de Estudios Históricos de la Masonería Española, España)

Kyle Jackson (University of California Los Angeles, United States)

Diseñador y administrador $W e b$ :

Luis Martín Valverde Alfaro (Universidad de Costa Rica, Costa Rica)

Editor:

Yván Pozuelo Andrés (IES Universidad Laboral de Gijón, España)

Correo electrónico: yvan@edu.xunta.es

Director:

Ricardo Martínez Esquivel (Universidad de Costa Rica, Costa Rica)

Correo electrónico: ricardo.martinezesquivel@ucr.ac.cr

Dirección Web: rehmlac.com/

Correo electrónico: info@rehmlac.com

Apartado postal: 243-2300 San José, Costa Rica 


\section{Citado en:}

Academia.edu

Aladin. WRLC. Libraries Catalog

AFEHC. Asociación para el Fomento de los Estudios Históricos en Centroamerica

Biblioteca de Georgetown

CRICCAL, Université Sorbonne Nouvelle Paris 3

CERGE EI. Portál elektronických časopisů. Univerzita Karlova v Praze

Departamento de Filosofía de la Universidad Centroamericana "José Simeón Cañas"

Dialnet, Universidad de la Rioja

Directorio y recolector de recursos digitales del Ministerio de Cultura de España

DOAJ. Directory of Open Access Journals

e-revist@s. Plataforma Open Access de Revistas Científicas Electrónicas

Freemasonry and Civil Society Program at UCLA

Fudan University Library Academic Resource Portal

Google académico

Institute for the Study of the Americas at University of London

Latindex (UNAM)

Latindex.ucr. Repositorio de revistas de la Universidad de Costa Rica

Library Catalogue of University of South Australia

Museo Virtual de la Historia de la Masonería de La UNED

Nuevo Mundo. Mundos Nuevos

REDIAL. Red Europea de Información y Documentación sobre America Latina

SciELO - Scientific Electronic Library Online

Sherpa/Romeo

SID. Sistema Integrado de Documentación. Universidad Nacional de Cuyo

Toronto Public Library

UBO. Revues en ligne. Université de Bretagne Occidentale

Universia. Biblioteca de Recursos

University of Saskatchewan Library

University of Wiscosin-Madison Libraries

Western Theological Seminary. Beardslee Library Journals 


\title{
"La participación político-partidista de la Iglesia: El Partido Unión Católica y sus estrategias de movilización política en el marco del conflicto entre la Iglesia Católica y el Estado liberal en Costa Rica (1889-1898)"
}

\author{
Esteban Sánchez Solano \\ Máster en Historia por la Universidad de Costa Rica, 2013. Profesor de la Escuela de Estudios Generales de la \\ Escuela de Historia de la Universidad de Costa Rica. \\ Correo electrónico: marhabanbikun@gmail.com
}

El investigador Esteban Sánchez Solano ha defendido una Tesis de Maestría en Historia, cuyo título es: "La participación político-partidista de la Iglesia: El Partido Unión Católica y sus estrategias de movilización política en el marco del conflicto entre la Iglesia Católica y el Estado liberal en Costa Rica (1889-1898)", en la Universidad de Costa Rica en Costa Rica, el 30 de agosto del 2013.

Esteban Sánchez Solano nació en 1980 en la ciudad de Cartago, Costa Rica.

El autor nos ha concedido la siguiente entrevista.

\section{¿Cuáles fueron los motivos que le llevó a enfocar su investigación hacia la Masonería?}

Mi tema de tesis no estaba estrictamente enfocado en la Masonería. No obstante, fueron recurrentes en el periodo de estudio escogido (la segunda mitad del siglo XIX) las referencias hacia la Masonería o a miembros de logias masónicas. Por ello, llegué a la conclusión que este tema debía tener un espacio importante en la tesis, siempre y cuando no sobrepasara mi tema central (la conformación de un partido católico, con apoyo de la Iglesia católica de Costa Rica, durante el periodo denominado liberal de la historia del país).

Sin embargo, debo dejar claro que fue la relación profesional cercana con el colega Ricardo Martínez Esquivel, cuya tesis de maestría versó sobre un estudio de la masonería desde la teoría de redes, ${ }^{1}$ lo que estimuló que asumiera con mayor preocupación, desde el punto de vista historiográfico, el tema de la Masonería. Al estar ambos elaborando la tesis simultáneamente, hubo la posibilidad de estar intercambiando continuamente información, observaciones, fuentes y críticas a los trabajos que cada uno estaba desarrollando.

De esta manera, la revisión de la literatura sobre la Masonería escrita en Costa Rica y en otras latitudes, fueron ganando un mayor peso del que inicialmente yo le había dado y, en consecuencia, en el tema de investigación propuesto. Esto me dejó una enseñanza en términos de investigación que espero recoger en mis futuros trabajos: la exhaustividad en la revisión de

\footnotetext{
${ }^{1}$ La cual finalmente tuvo el siguiente título: Ricardo Martínez Esquivel. "Masones y Masonería en la Costa Rica de los albores de la Modernidad (1865-1899)". Tesis de Maestría en Historia, Universidad de Costa Rica, 2012.
} 
la literatura para no dejar vacíos que puedan afectar el resultado final de cualquier investigación en el futuro. Ese itinerario debe contemplarse con mayor ahínco en el mundo académico.

\section{¿Cuáles fueron las fuentes que ha utilizado?}

En este respecto, el punto clave para mí era entender lo que significaba para la Iglesia la presencia de la Masonería en la sociedad y, en particular, dentro del ámbito político, producto de la construcción ideológica que hizo la institución eclesiástica desde el siglo XVIII en Europa de una "confabulación" masónica para tomar el poder. Esto, desde el punto de vista de las fuentes, me llevó a buscar la visión de la Iglesia sobre la masonería en parte de su producción oficial (cartas pastorales, circulares al clero, folletos, panfletos, hojas sueltas, periódicos) así como en otro tipo de documentación cuya característica común fuese en todo momento la presencia "real" o "imaginaria" de la Masonería en la sociedad costarricense (correspondencia, informes de parroquias, memorandos, entre otras).

Lo relevante aquí fue la explotación sistemática que pretendí de los archivos eclesiásticos para este cometido. En general estos archivos, al menos en el caso de Costa Rica, son desestimados por una parte de la historiografía. Existen diversos temas que, sin ser estrictamente sobre fenómenos religiosos, pueden ser profundizados utilizando sistemáticamente los archivos eclesiásticos, como lo hizo la historiografía costarricense durante las décadas de 1970 y 1980 respecto a los estudios demográficos.

Volviendo al tema original, yo pude encontrar, tras meses de búsqueda y mucha paciencia, diversas expresiones sociales referidas a la Masonería. El punto que siempre estuvo detrás para utilizar estas fuentes, fue en qué medida esta vasta información que encontré me podía dar pistas para comprender comportamientos ligados al escenario político partidista que se venía gestando en Costa Rica a partir de la década de 1880. De esta manera, a la hora de plantear el peso de la Masonería en el ámbito político me acercaba desde los propios actores involucrados en diferentes contextos antes que asumir ideas vagas sobre la Masonería. De esta manera, la Masonería se convertía en un actor con cara pero, al mismo tiempo, tomando distancia de la "tesis" confabuladora ${ }^{2}$ que circula ampliamente por la sociedad desde diversos ámbitos culturales.

\section{¿Cuáles fueron las principales dificultades con las que se ha encontrado? ¿Cómo las ha superado?}

La primera podría considerarse como de orden metodológico, en tanto la manera de resolver el uso que se puede hacer de las fuentes para llegar a conclusiones válidas. En el caso de la Masonería, este es un tema que se presta (como pocos) al uso de ideas preconcebidas que son disfrazadas de análisis, tanto por los actores involucrados del proceso estudiado como por elaboraciones historiográficas posteriores. Ya que la postura de ser masón o no permea las

\footnotetext{
${ }^{2}$ La cual, valga aclarar, es una temática de análisis per se.
} 
investigaciones. Esto, en buena medida, fue generado por la actitud intransigente de la Iglesia hacia este espacio asociativo. En este sentido, a la hora de enfrentar el análisis de las fuentes referidas a la Masonería, lo que busqué fue definir claramente las condiciones socio-culturales en que estas fueron elaboradas, que me permitieran dar cuenta de su vínculo con el ámbito político en tanto la Iglesia lo refería como "enemigo" político o partidista.

Aunque esto último señalado puede sonar obvio en la investigación histórica, es recurrente aún en nuestros días lecturas simplistas o con poca crítica de las fuentes. Por ello, el principio de procedencia de la fuente (criterio asumido entre los archivistas) debe ser considerado por el historiador como básico en su labor de investigación. De esta manera, al contemplar el recorrido que la misma fuente ha tenido (institucional y social), puede descubrirse aspectos relevantes de las motivaciones de los actores, aunque la información presente en las fuentes no se le puede considerar como "cierta" en cualquier circunstancia. Esta discusión heurística, aunque ha avanzado considerablemente en la historiografía costarricense, no es tan visible en la producción escrita, aunque existe en las aulas.

\section{¿Cuáles fueron las principales problemáticas históricas que su trabajo ha resuelto?}

Una fundamental es que el estudio de lo institucional puede dar otros frutos si no se asume su estructura de una forma rígida. Esto es clave ya que se sigue reproduciendo muchos estereotipos de la relación entre la Iglesia y el Estado y el papel que pudo tener la masonería en la realidad social durante el siglo XIX. Al mismo tiempo, ofreció un acercamiento diferente a las relaciones de poder a partir de la utilización del análisis de redes como estrategia metodológica.

De esta manera, las respuestas de las acciones de los actores van a ser dadas por el universo de sus relaciones dentro de la estructura y no una acción mecánica de la estructura hacia los actores de forma vertical. Esto permite constatar que los estudios sobre el poder, al menos en Costa Rica, adolecen todavía de una visión más compleja de los núcleos de las motivaciones de los actores, ya que sus análisis se dirigen en alguna medida a responder solo por contexto lo que buscan en el fondo. Los trabajos de Eduardo Madrigal, historiador que ha utilizado sistemáticamente la teoría de redes, escapa a estos límites. Otro que ha aportado desde la historiografía, aunque aún le falta desarrollar un trabajo empírico más acucioso, es Hugo Vargas González, quien se ha concentrado en el espacio político-electoral (valga aclarar que ninguno ha trabajado directamente la masonería, pero sus trabajos permiten pensar, desde lo teórico y metodológico, posibilidades para acercarse a la masonería, al menos desde la historia del poder).

\section{¿Por favor, podría resumir la esencia de su Tesis en dos líneas?}

La tesis buscó comprender la manera en que la Iglesia pensó estrategias para solventar sus conflictos con el Estado liberal del siglo XIX acudiendo a diversos espacios que estaban siendo redefinidos por la construcción de la nueva comunidad política. Esto fue llevado a cabo, entre otras cosas, acudiendo a escenarios vitales de la reproducción del poder (no solo 
político, sino también de otro tipo como el cultural, social, de las ideas, el electoral). No obstante, mi propuesta tomó como punto de partida el poder centrado en la autoridad política.

La formación del Partido Unión Católica fue el corolario de esa estrategia de la Iglesia, por lo demás compleja ya que la Iglesia en su interior tiene una dinámica que estaba muy lejos de ser monolítica. Por ello, el Unión Católica se convirtió en una ventana idónea para definir el perfil que estaba teniendo la dinámica institucional en Costa Rica, incluida su relación con la masonería la cual, como dije anteriormente, era una de las temáticas derivadas de la tesis, ya que el periodo del Partido Unión Católica coincide con la presencia "real" de la masonería en la sociedad costarricense.

\section{¿Cuáles fueron las lecciones, a todos los niveles, personal y profesional, que usted ha deducido de su experiencia investigativa?}

Desde lo personal, la posibilidad de compartir con varios colegas con temas afines o cercanos al mío permitió aclarar a través del proceso de investigación las posibilidades y los límites del trabajo que propuse. Este contacto continuo debe quedar con mayor huella institucional y escrita de la que existe, ya que la investigación, aunque se aprende haciendo, necesita en muchas ocasiones de estímulos exógenos al proceso individual de construir el trabajo que durará varios años.

Respecto a lo profesional, pude observar que las posibilidades que tienen los proyectos de investigación de ser difundidos dependen en buena medida de que se generen espacios para hacerlo. Esto puede sonar obvio cuando se puede señalar que existen escenarios para eso, pero no todos los temas tienen la misma vigencia. Aquí entra en juego incluso los gustos, los cuales pueden cerrarle puertas a temas relevantes pero que no logran una acogida por barreras personales o institucionales. Por ello, revistas como REMHLAC cumplen un papel fundamental en esto, ya que estimulan a investigadores a dejar huella de lo que fue su experiencia de investigación.

\section{¿Ahora, cuáles son sus proyectos profesionales?}

Estoy interesado en profundizar el recorrido de los fenómenos religiosos en la construcción de la modernidad. He querido entrar de lleno en la re-visión de la conformación de la religiosidad a partir del siglo XVI, con la intención de desarrollarlo de una manera cronológica. Esto último me ha llevado a buscar nuevas vetas dentro del estudio de los fenómenos religiosos y su papel no solo como "legitimadores" de la transformación radical que inicia con la expansión europea, sino como parte del núcleo creador de nuevas realidades.

De alguna manera, quiero seguir una línea que autores como Serge Gruzinski, Svetan Todorov, Elisabetta Corsi o Benedetta Albani, entre otros, han desarrollado de manera novedosa sobre lo religioso en la modernidad a partir de la década de 1980 y que, en el último lustro ha tenido un gran impulso. Aquí la masonería puede entrar como posible objeto de estudio, a partir de los fenómenos relacionados con ella del siglo XVIII en adelante, en tanto lo que significaba su reto a las fuentes de autoridad religiosas o seculares, asumiéndolo desde 
un análisis historiográfico, pero eso dependerá del propio desarrollo de mi actividad investigativa.

\section{¿Quisiera destacar algún aspecto que no se ha contemplado y que usted consideraría digno de reseñar?}

La necesidad de estudiar los fenómenos históricos de otra manera. El vínculo entre diversas perspectivas (social, política, económica, entre otras) debe ser mucho más explícito entre los investigadores. No obstante, eso se debe definir desde el objeto de investigación que se ha propuesto sin cerrar la puerta desde el principio que lo que se está haciendo es historia política o historia social, por poner un ejemplo. Esto no puede ser el leitmotiv de la investigación histórica. Más bien, encontrar canales que nutran diversas perspectivas dentro de los proyectos de investigación que se propongan.

Cuando inicié la investigación asumí el falso problema de definir de antemano si lo que iba a hacer era historia política o historia eclesiástica, entre otras etiquetas. Al final me di cuenta que esto no era lo que definía exclusivamente mi trabajo. En la tesis realicé análisis de redes, de las ideas, del poder. No obstante, al final lo relevante era acercarme a una expresión particular que fue un partido político católico y, en lo referente a la Masonería, el papel que tuvo en tanto los actores involucrados en la política que eran vistos como enemigos políticos por el partido católico, así como el peso desde el imaginario que le dio la Iglesia como una amenaza al mundo católico en todas sus expresiones.

Esta entrevista se efectúo de forma telemática el 15 de septiembre de 2014.

Autores de la entrevista: Ricardo Martínez Esquivel e Yván Pozuelo Andrés, Director y Editor de REHMLAC. 